\title{
Development of immune and microbial environments is independently regulated in the mammary gland
}

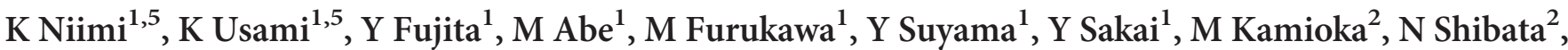

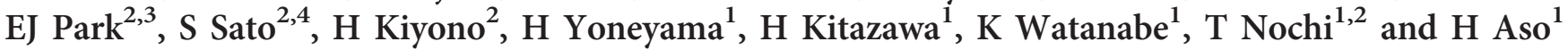

Breastfeeding is important for mammals, providing immunological and microbiological advantages to neonates, together with the nutritional supply from the mother. However, the mechanisms of this functional diversity in the mammary gland remain poorly characterized. Here, we show that, similar to the gastrointestinal tract, the mammary gland develops immune and microbial environments consisting of immunoglobulin A $(\operatorname{IgA})$ and the microflora, respectively, both of which are important for protecting neonates and the mother from infectious diseases. The IgA production and microflora development are coordinated in the gastrointestinal tract but seem to be independently regulated in the mammary gland. In particular, the chemokine (C-C motif) ligand 28 and poly-Ig receptor, crucial molecules for the IgA production in milk, were expressed normally in germ-free lactating mice but were almost undetectable in postweaning mothers, regardless of the microflora presence. Our findings offer insights into potentially improving the quality of breastfeeding, using both immunological and microbiological approaches.

\section{INTRODUCTION}

The mammary gland is characterized by unique physiological features in the developmental process as it is intensely influenced by mammotropic hormones associated with pregnancy and lactation. ${ }^{1}$ Unlike other exocrine organs such as the salivary gland, which constitutively produces saliva, the mammary gland is only developed during a limited period in life to produce milk. ${ }^{2}$ Antibodies contained in the maternal milk play an important role in protecting neonates from bacterial and viral infections. ${ }^{3}$ In humans, immunoglobulin A (IgA) is the dominant immunoglobulin (Ig) subclass in milk, ${ }^{4}$ and it is secreted locally by plasma cells that migrate into the mammary gland, possibly from the gut-associated lymphoid tissue. ${ }^{5}$ The chemokine (C-C motif) ligand 28 (CCL28), also known as the mucosa-associated epithelial chemokine, has a critical role in recruiting IgA-producing plasma cells into the mammary gland. Blockage of CCL28 function by the treatment with anti-CCL28 antibodies or deletion of the $\mathrm{C}-\mathrm{C}$ chemokine receptor type 10 (CCR10), which is a receptor for CCL28, prevents the recruitment of IgA-producing plasma cells into the mammary gland, resulting in the abolishment of IgA production in milk. ${ }^{6,7}$ The polymeric Ig receptor (pIgR) is also involved in IgA production in milk. Initially expressed at the basal surface of mammary epithelial cells, pIgR recognizes IgA to transport it toward the apical surface for secretion into mammary alveoli. ${ }^{8}$ Therefore, the recruitment of plasma cells into the mammary gland through CCL28/CCR10 and the transportation of IgA into mammary epithelial cells via pIgR are indispensable for IgA production in milk. ${ }^{9}$

Recent advances in microbiome analysis have opened new avenues for microbial research as they allow studying unculturable microorganisms that are impossible to isolate by classical, culture-dependent methods. ${ }^{10}$ It has been demonstrated that using these techniques bacterial communities are

${ }^{1}$ International Education and Research Center for Food and Agricultural Immunology, Graduate School of Agricultural Science, Tohoku University, Miyagi, Japan. ${ }^{2}$ International Research and Development Center for Mucosal Vaccine, Institute of Medical Science, University of Tokyo, Tokyo, Japan. ${ }^{3}$ Department of Molecular Pathobiology and Cell Adhesion Biology, Graduate School of Medicine, Mie University, Mie, Japan and ${ }^{4}$ Mucosal Vaccine and Adjuvant Project, Research Institute for Microbial Diseases, Osaka University, Suita, Japan. Correspondence: T Nochi (nochi@m.tohoku.ac.jp)

${ }^{5}$ Equivalent co-first authors. 
developed in human milk, ${ }^{11}$ although the origin is still unclear. Bacteria present in the infant's oral cavity or on mother's skin were traditionally suspected of milk contamination through breastfeeding; ${ }^{12}$ however, recent literature has provided a hypothesis that such bacteria may be translocated from the maternal gut. ${ }^{13,14}$ Given that the presence of microflora enhances resistance to colonization of pathogens and IgA is likely to play a particularly important role in the frontline defense barrier in mucosal tissues, the improvement of quality of microflora and maternal IgA in the mammary gland could be an ideal approach to not only maintain the satisfactory breastfeeding but also control an outbreak of a bacterial infection that causes inflammation (mastitis). ${ }^{13}$

Here, we show that the microbial diversity can be detected in the mouse mammary gland just after parturition; however, there is a time lag before the IgA production is initiated in the tissue. CCL28 and PIgR were both expressed normally in the mammary gland of germ-free lactating mice that lacked the microflora, whereas the expression of CCL28 and pIgR was almost undetectable in postweaning mothers. These results indicate that the immune and microbial environments are developmentally established in the mammary gland.

\section{RESULTS \\ IgA levels in milk gradually increase during lactation in mice}

To first confirm that IgA is the most abundant Ig subclass in mouse milk throughout the lactation period, we sampled the stomach contents from 0-, 7-, and 14-day-old pups (0, 1, and 2 weeks after the parturition) and performed a chronological study to determine the concentrations of three major Ig subclasses (i.e., IgA, IgG, and $\operatorname{IgM}$ ) by an enzyme-linked immunosorbent assay (ELISA). We found that IgA was the most abundant Ig, whereas IgG and IgM were barely detected, regardless of the lactation time point (Figure 1). Consistent with previous findings, ${ }^{14}$ the IgA level in milk gradually increased during lactation, and the concentration was the highest 2 weeks after parturition and the lowest just after parturition (Figure 1).

\section{Suckling is essential for the recruitment of IgA-producing cells into the mammary gland}

IgA found in mucosal secretions is produced by plasma cells that are locally accumulated in mucosal tissues. ${ }^{15,16} \mathrm{We}$ therefore examined the presence of IgA-producing cells in the mammary gland by histological analysis. Considering that the mammary gland is developed under the influence of hormones (e.g., estrogen) that are highly produced during pregnancy, ${ }^{1}$ we collected mammary gland samples over a long period throughout the reproduction cycle. When we initially performed hematoxylin and eosin staining of tissue sections of mammary glands obtained from nonpregnant nulliparous mice (8-10 weeks old), we found that mammary ducts were only developed around adipose tissue (Figure 2a). In contrast, development of alveolar buds was observed in the mammary gland analyzed at 13 days of gestation (1 week before the parturition), and alveoli promptly maturated after the

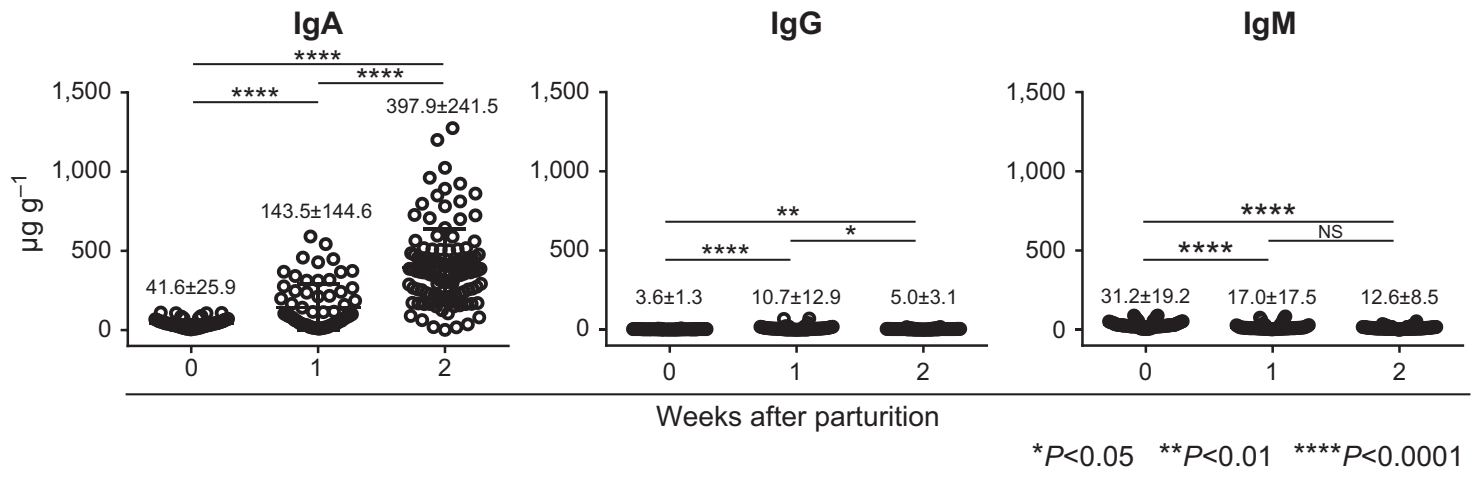

Figure 1 Alteration of immunoglobulin (Ig) levels in milk after parturition. Concentrations of three immunoglobulin subclasses (IgA, IgG, and IgM) in milk obtained just after birth $(n=100)$ and 1 week $(n=68)$ and 2 weeks $(n=112)$ later from stomach contents of pups were measured by enzyme-linked immunosorbent assay (ELISA). Among the three immunoglobulin subclasses, IgA was the dominant immunoglobulin in milk throughout the experimental period ( 2 weeks after the parturition). The lgA levels gradually but significantly increased after the parturition. The " $n$ " indicates the number of pups that were used for collecting stomach contents containing milk samples. The unit " $\mu \mathrm{g} \mathrm{g}^{-1}$ " on the $y$ axis indicates how much $\mu \mathrm{g}$ of antibody is included in $1 \mathrm{~g}$ of stomach content. ${ }^{*} P<0.05 ;{ }^{* *} P<0.01 ;{ }^{* * *} P<0.0001$; NS, not significant.

Figure 2 Morphological and immunological characteristics of the mammary gland throughout the reproduction cycle. (a) Mammary gland tissues were collected at 1 week before parturition $(n=5)$, just after parturition $(n=5)$, and 1 week $(n=5)$, 2 weeks $(n=5)$, and 6 weeks $(n=5)$ after parturition. Tissues were also collected 2 weeks after parturition from mice $(n=5)$ whose pups were excluded 1 week earlier. Nonpregnant nulliparous mice were used as a control group $(n=5)$. Development of mammary gland alveoli was initiated at a pregnancy stage and accelerated after the parturition. However, the tissue became atrophied immediately after weaning and was similar to that of nulliparous mice. Immunoglobulin A (IgA)-producing cells were distributed around mammary gland alveoli. (b) The number of IgA-producing cells gradually but significantly increased during lactation but decreased immediately after both forced and normal weaning. Few IgA-producing cells were found in pregnant and nulliparous mice. Scale bar $=50 \mu \mathrm{m}$. $\mathrm{HE}$, hematoxylin and eosin staining; IHC, immunohistochemistry. ${ }^{\star} P<0.05 ;{ }^{\star \star} P<0.01 ;{ }^{\star \star \star} P<0.001$. 
a

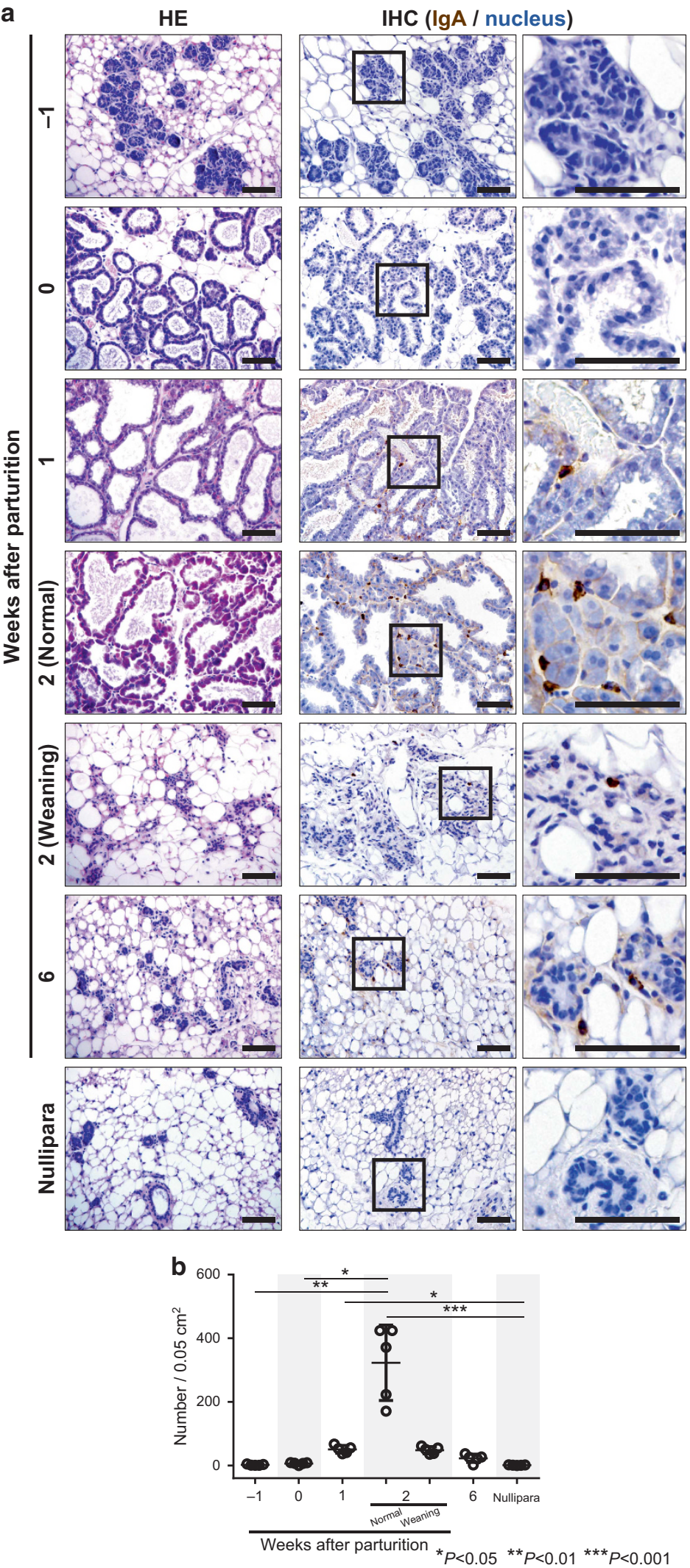


parturition (Figure 2a). It should be noted that the suckling signal was essential for the maintenance of mature alveoli because the tissue rapidly became atrophied within 1 week if pups were force-weaned, even though the mother was still within the usual 3-week lactation period (Figure 2a). Development of the mammary grand completely regressed after the normal weaning (Figure 2a). Based on the knowledge of morphological characteristics of the mammary gland throughout the reproduction cycle, we next performed immunohistochemical analysis to detect the presence of IgA-producing cells at each stage. Consistent with our findings shown in Figure 1, few IgA-producing cells were found in the mammary gland analyzed just after the parturition, whereas a large number of the cells were observed around mature alveoli in the mammary gland 2 weeks after parturition (Figure 2a,b). Importantly, only a few IgA-producing cells were detected in mammary glands of mice after both forced and normal weaning; however, these cells were almost undetectable when antenatal mice, including pregnant and nonpregnant nulliparous mice, were examined (Figure 2a,b). These results indicate that the suckling stimulation is essential for the maintenance of not only mature alveoli but also of IgAproducing cells in the mammary gland.

\section{Plasma cells fully differentiated from mature B cells are present in the mammary gland}

Mucosa-associated lymphoid tissues (MALTs) such as Peyer's patches contain naive lymphocytes, including mature B cells. ${ }^{15,17}$ Once B cells are activated in MALTs by antigenic stimulation, they induce the Ig class switching from IgM to IgA and differentiate into plasmablasts and to plasma cells that are predominantly localized in mucosal tissues (e.g., intestinal lamina propria). ${ }^{15,17}$ The inguinal lymph node is the draining lymph node of the mammary gland; ${ }^{16}$ however, its role in IgA production in the mammary gland is still under investigation. We therefore examined cells that were individually isolated from the mammary gland and inguinal lymph node to determine B-cell maturation and differentiation in the mammary immune system. When we analyzed the cells by flow cytometry after staining with anti-IgA and anti-B220 antibodies, we found that $\operatorname{IgA}{ }^{+} \mathrm{B}_{2} 20^{-}$cells, which correspond to plasma cells, were highly accumulated in the mammary gland 2 weeks after parturition. Consistent with the findings obtained by immunohistochemistry (Figure 2), the number of plasma cells in the mammary gland gradually increased after parturition and rapidly decreased after weaning (Figure 3a). In nulliparous mice, few plasma cells were detected in the mammary gland (Figure 3a). $\operatorname{IgA}^{-} \mathrm{B}_{22} 0^{+}$cells, which correspond to mature $\mathrm{B}$ cells, were almost undetectable in the mammary gland throughout the reproduction cycle (Figure 3a). In contrast, a large number of mature B cells were constantly found in the inguinal lymph node, but plasma cells were barely detected throughout the experimental period (Figure 3a). When we calculated the absolute numbers of the total cells isolated, including IgA ${ }^{-} \mathrm{B}_{2} 20^{+}$mature $\mathrm{B}$ cells and $\mathrm{IgA}^{+} \mathrm{B}_{220^{-}}$plasma cells, the change trends were similar for the total cells and plasma cells present in the mammary gland (Figure 3b). Given that the total cells also include alveolar epithelial cells, which are developed in mammary gland alveoli, these results indicate that in accordance with the development of mammary gland alveoli, immune cells, including plasma cells, can migrate into the mammary gland. In the inguinal lymph node, no significant increase or decrease of the absolute numbers of total cells, mature B cells, or plasma cells was seen throughout the experimental period (Figure $\mathbf{3 b}$ ). It should be noted that unlike Peyer's patches that play an important role in initiating IgA production in the gut immune system (Supplementary Figure S1 online), a differentiation process from mature $\mathrm{B}$ cells into $\mathrm{IgA}^{+} \mathrm{B}_{22}{ }^{+}$plasmablasts that underwent immunoglobulin class switching into $\operatorname{IgA}$ after antigenic stimulation were not found in the inguinal lymph node (Figure 3a). However, it potentially acts as a gateway for pathogens that infected locally via nipples (Supplementary Figure S2). These results suggest that the inguinal lymph node may not be initiatively involved in maternal IgA production unless local infection occurs in the mammary gland.

\section{Indigenous flora in the mammary gland does not dramatically change throughout the lactation period}

Because the IgA production in the mammary gland gradually increased during lactation, we assumed that it might be influenced by microbial stimulation through breastfeeding. To address this hypothesis, we performed a quantitative PCR analysis using genomic DNA extracted from mammary glands of mice immediately or 2 weeks after the parturition. We took the advantage of the tuf gene to amplify it by quantitative PCR because the tuf gene is highly conserved among bacterial species and only one or two copies are present on the chromosome. ${ }^{18}$ When we tested genomic DNA extracted from a total of 10 mice, of which 5 mice were just after parturition and 5 were 2 weeks after parturition, the $t u f$ gene was amplified in all samples (Figure 4a). The levels of expression of the tuf gene in the mammary gland varied widely among the mice at 2 weeks after parturition, but no statistical difference was observed between the two groups (Figure 4a). Subsequent analysis of the $16 \mathrm{~S}$ ribosomal RNA gene by next-generation sequencing revealed that the diversity of the microbiota was established in the mammary gland regardless of the time after parturition (Figure 4b). Using the QIIME and R software to perform microbial community and statistical studies, we compared the microbiotas between the two groups by a principal component analysis to create a biplot (Figure 4c) and used the Wilcoxon signed-rank test to obtain $P$ values. Correspondingly, either Student's or Welch's $t$-test was carried out in accordance with the homoscedasticity examined by F-tests. Based on the data obtained by the Wilcoxon signed-rank test or $t$-tests, we noticed that there were statistically significant differences $(P<0.05)$ between the two groups in the frequency of 29 or 26 species, respectively, of the 780 species that were identified by nextgeneration sequencing (Supplementary Table S1). However, when the Benjamini-Hochberg procedure for controlling the false discovery rate was performed to obtain $q$-values, 


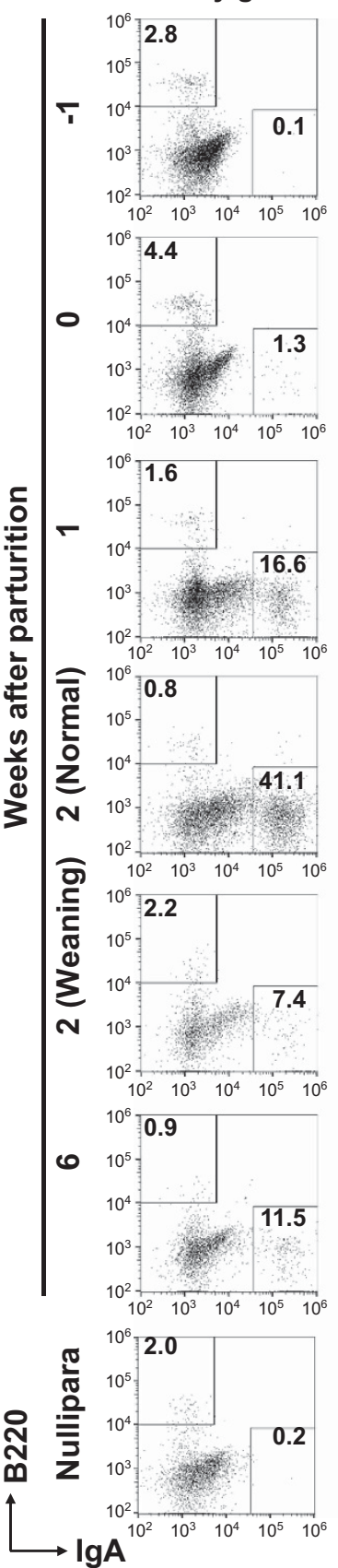

b
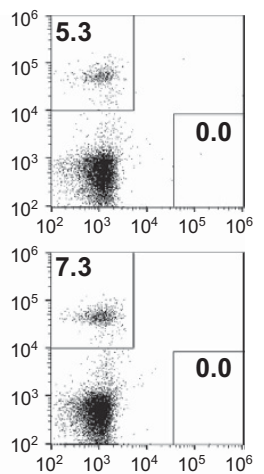

$1 0 ^ { 6 } \longdiv { 8 . 1 }$

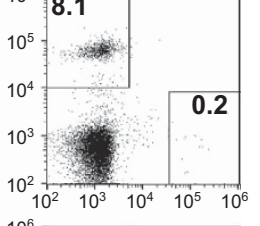

$10^{6} \overline{\mathrm{Z}} \mathbf{7 . 2}$

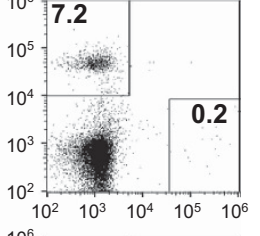

$10^{6} \overline{10.4}$
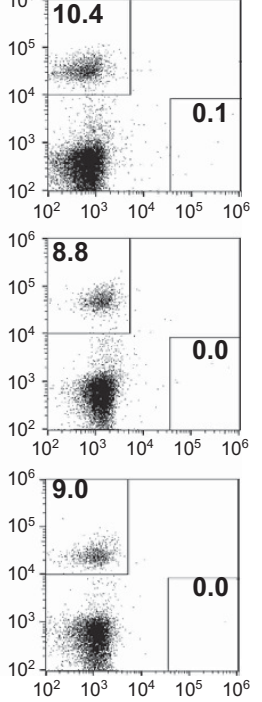

Mammary gland

Inguinal lymph node

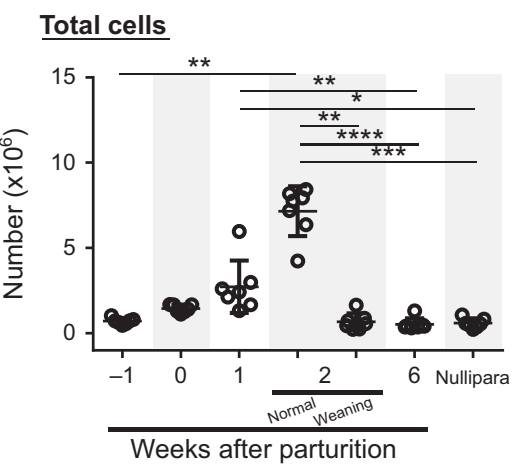

Mature B cells $\left({\left.\mathrm{B} 220^{+} \lg \mathrm{A}^{-}\right)}^{-}\right.$

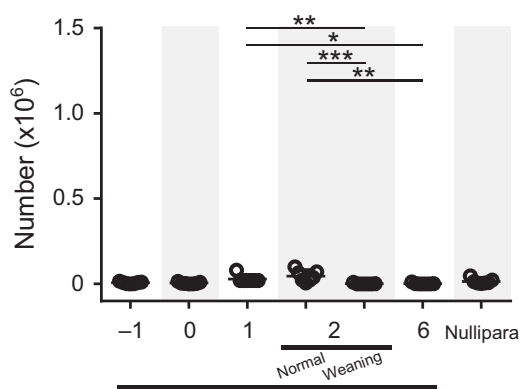

Weeks after parturition

Plasma cells (B220- $\left.\lg \mathrm{A}^{+}\right)$

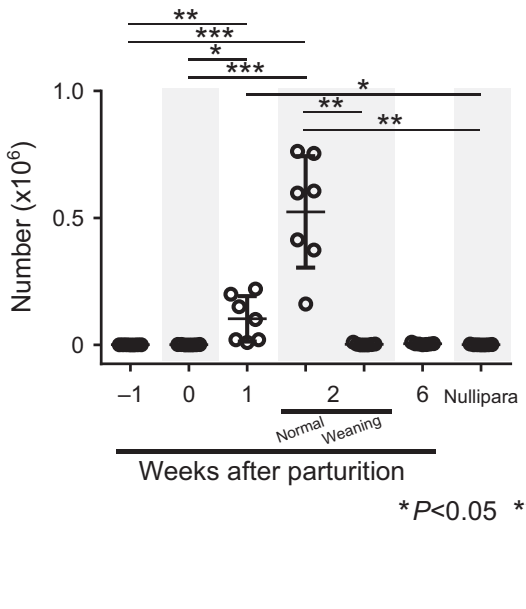

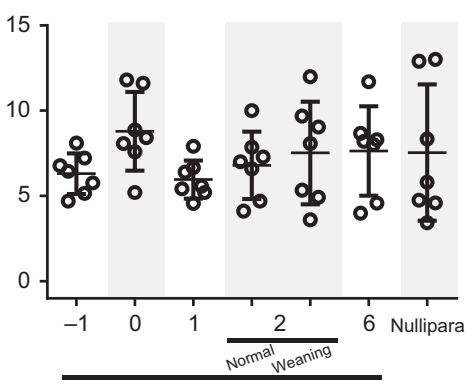

Weeks after parturition

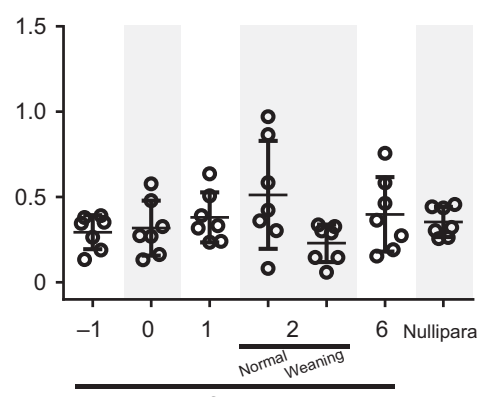

Weeks after parturition

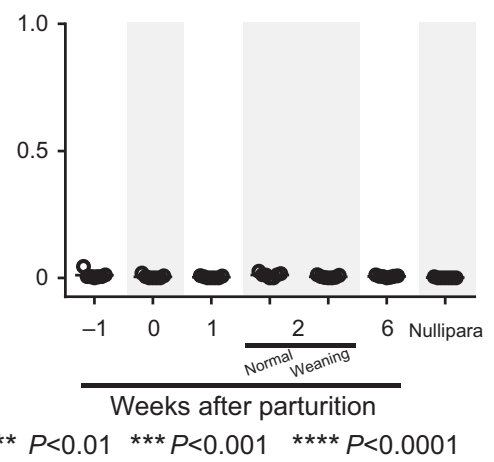

Figure 3 B-cell differentiation in the mammary gland. (a) To isolate mononuclear cells, mammary glands and inguinal lymph nodes were individually harvested at 13 days of gestation $(n=7)$, just after parturition $(n=7)$, and 1 week $(n=7), 2$ weeks $(n=7)$, and 6 weeks $(n=7)$ after parturition. Tissues were also collected 2 weeks after parturition from mice $(n=7)$ whose pups were force-weaned 1 week earlier. Nonpregnant nulliparous mice were used as a control group $(n=7)$. In the mammary gland, IgA ${ }^{+} \mathrm{B} 220^{-}$plasma cells were remarkably found 2 weeks after parturition, whereas $\lg \mathrm{A}^{-} \mathrm{B} 220^{+}$mature $\mathrm{B}$ cells were a minor cell population in all groups tested. In the inguinal lymph node, $\lg \mathrm{A}^{-} \mathrm{B}_{2} 2 \mathrm{O}^{+}$mature $\mathrm{B}$ cells were constantly found, whereas $\operatorname{IgA}{ }^{+} \mathrm{B}_{220^{-}}$plasma cells were hardly detected. (b) Absolute numbers of $\lg \mathrm{A}^{-} \mathrm{B}^{2} 20^{+}$mature $\mathrm{B}$ cells and $\lg \mathrm{A}^{+} \mathrm{B}^{-220^{-}}$plasma cells were calculated based on the number of total cells isolated and the frequency of each cell population in a flow cytometry profile. Because mammary gland alveoli are developed after the parturition, a large number of mononuclear cells were isolated 2 weeks after the parturition $\left(7.16 \pm 1.46 \times 10^{6}\right.$ cells per fourth and fifth mammary glands). Importantly, the highest number of $\operatorname{lgA}{ }^{+} \mathrm{B} 220^{-}$plasma cells was also observed 2 weeks after the parturition $\left(0.52 \pm 0.22 \times 10^{6}\right.$ cells per fourth and fifth mammary glands). The numbers of total cells, $\operatorname{lgA}{ }^{-} \mathrm{B}_{2} 2 \mathrm{O}^{+}$mature $\mathrm{B}$ cells, and $\operatorname{lgA}{ }^{+} \mathrm{B}_{2} 2 \mathrm{O}^{-}$plasma cells in the inguinal lymph node did not vary throughout the reproduction cycle. ${ }^{*} P<0.05 ;{ }^{* *} P<0.01 ;{ }^{* * *} P<0.001 ;{ }^{* * * *} P<0.0001$.

all null hypotheses were suspended $(q>0.05)$ (Supplementary Table S1). These results indicated that the microbial diversity did not dramatically change in the mammary gland throughout the lactation period. Thus, it might have no influence on the elevation of IgA production in the mammary gland during 2 weeks after parturition. 
a
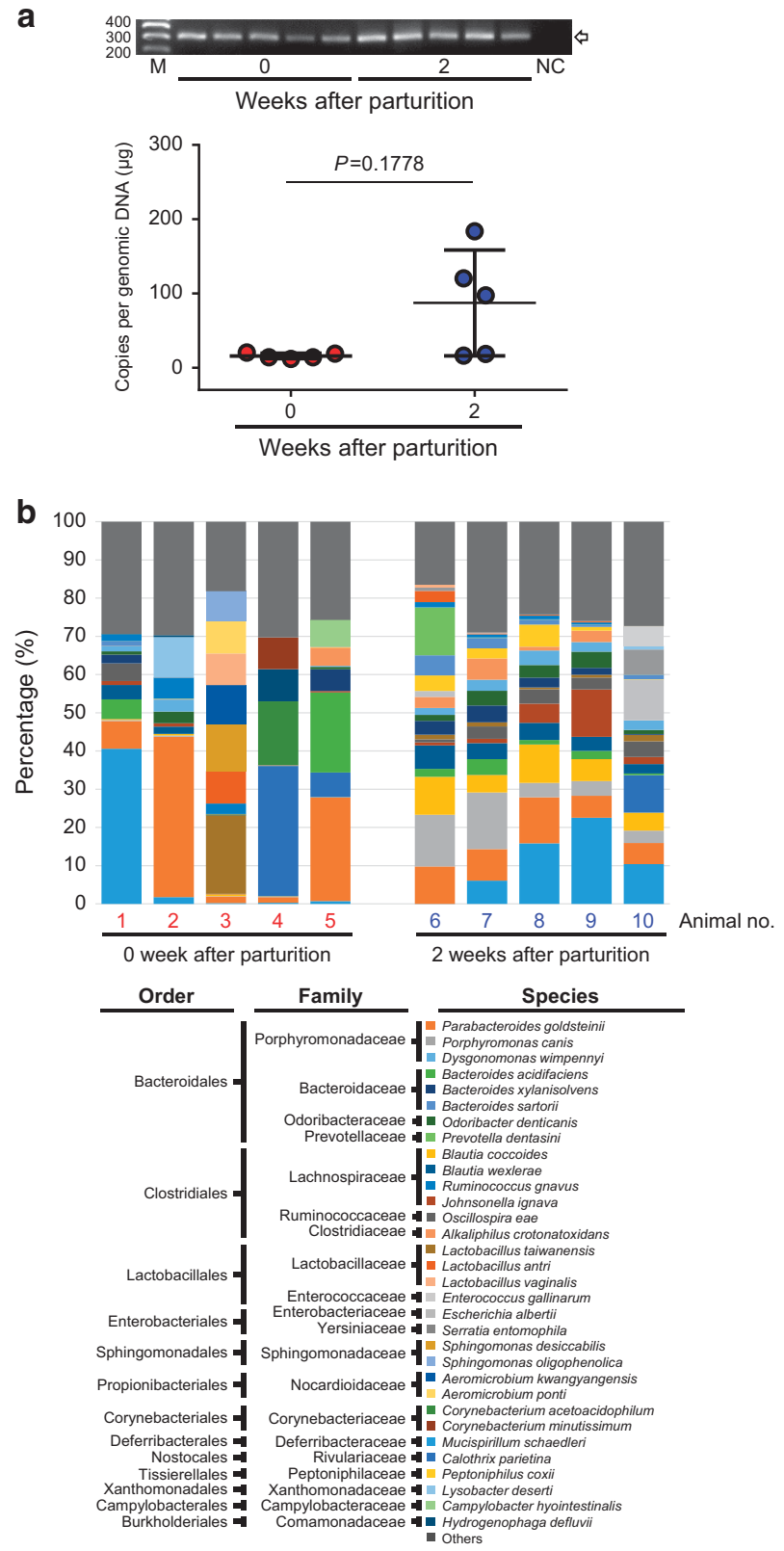

c

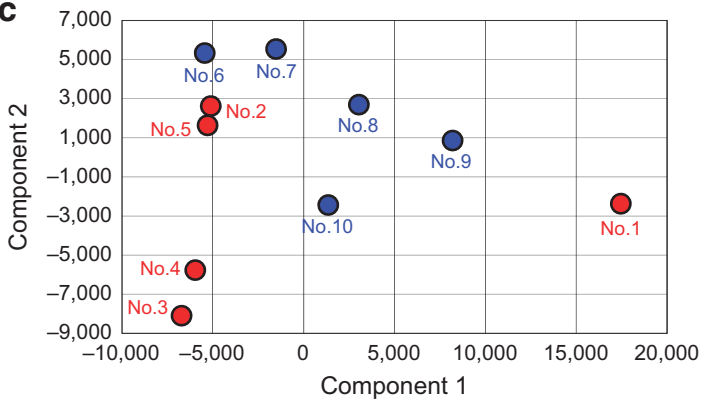

0 week after parturition

2 weeks after parturition
Recruitment of IgA-producing plasma cells into the mammary gland and transportation of secreted IgA into mammary alveoli are controlled endogenously

Mammary epithelial cells express CCL28 and pIgR, both of which play important roles in the IgA production in milk. Specifically, CCL28 has a chemotactic activity necessary to recruit IgA-producing plasma cells into the mammary gland. pIgR, which is initially expressed on the basal membrane of mammary epithelial cells, binds to the IgA secreted in the stroma and transports it into mammary alveoli. Given that the IgA level in milk gradually increased during lactation, without a dramatic change of microbial diversity in the mammary gland, we next evaluated the actual involvement of the microbiota in the induction of gene expression related to the IgA production in the mammary gland (i.e., expression of CCL28 and pIgR). Consistent with the findings showing a gradual increase of IgAproducing plasma cells in the mammary gland after parturition (Figures 1-3), the expression level of CCL28 in the mammary gland of conventional mice also gradually increased and reached a maximum 2 weeks after parturition (Figure 5a). Importantly, when we compared germ-free and conventional mice, both at 2 weeks after parturition, the levels of CCL28 were comparable; however, the level of CCL28 was almost undetectable in the conventional mice after forced weaning (Figure 5a). In nonlactating mice (i.e., pregnant, after normal weaning, and nulliparous mice), a very low level of CCL28 expression or no expression was found (Figure 5a). The expression patterns of $\mathrm{pIgR}$ and IgA in the mammary gland were found to be similar to that of CCL28 throughout the experiments conducted in this study (Figure 5a). Similar to germ-free mice, mice that mostly affect gut microorganisms by freely administered antibiotics also produce sufficient levels of CCL28 and pIgR in the mammary gland (Supplementary Figure S3). It should be noted that the expression levels of both CCL28 and pIgR were positively correlated with those of $\alpha$-, $\beta$-, and $\kappa$-casein, which are all well known as major milk proteins, when all data obtained from each group were combined for each gene and used for correlation studies (Figure 5a,b). Taken together, the results indicate that lactation-related endogenous factors (e.g., mammotropic hormones), rather than exogenous factors (e.g., microflora), may participate in the development of the mammary gland immune system to produce maternal antibodies.

Figure 4 Development of microflora in the mammary gland. (a) DNA was extracted from the mammary gland just after the parturition $(n=5)$ and 2 weeks later $(n=5)$ to demonstrate the presence of the microbiota and compare the diversity. The bacterial gene tuf was commonly detected in the mammary gland immediately after the parturition and 2 weeks later. There was no significant difference in the copy numbers between the two groups. (b) Metagenomics analysis using a next-generation sequencer identified 780 bacterial species commonly present in mammary glands of a total of 10 mice used. A diverse microbiota was found in the mammary gland regardless of the postparturition period analyzed. (c) A principal component analysis was performed to compare the microbiotas between the two groups and to create the biplot. 

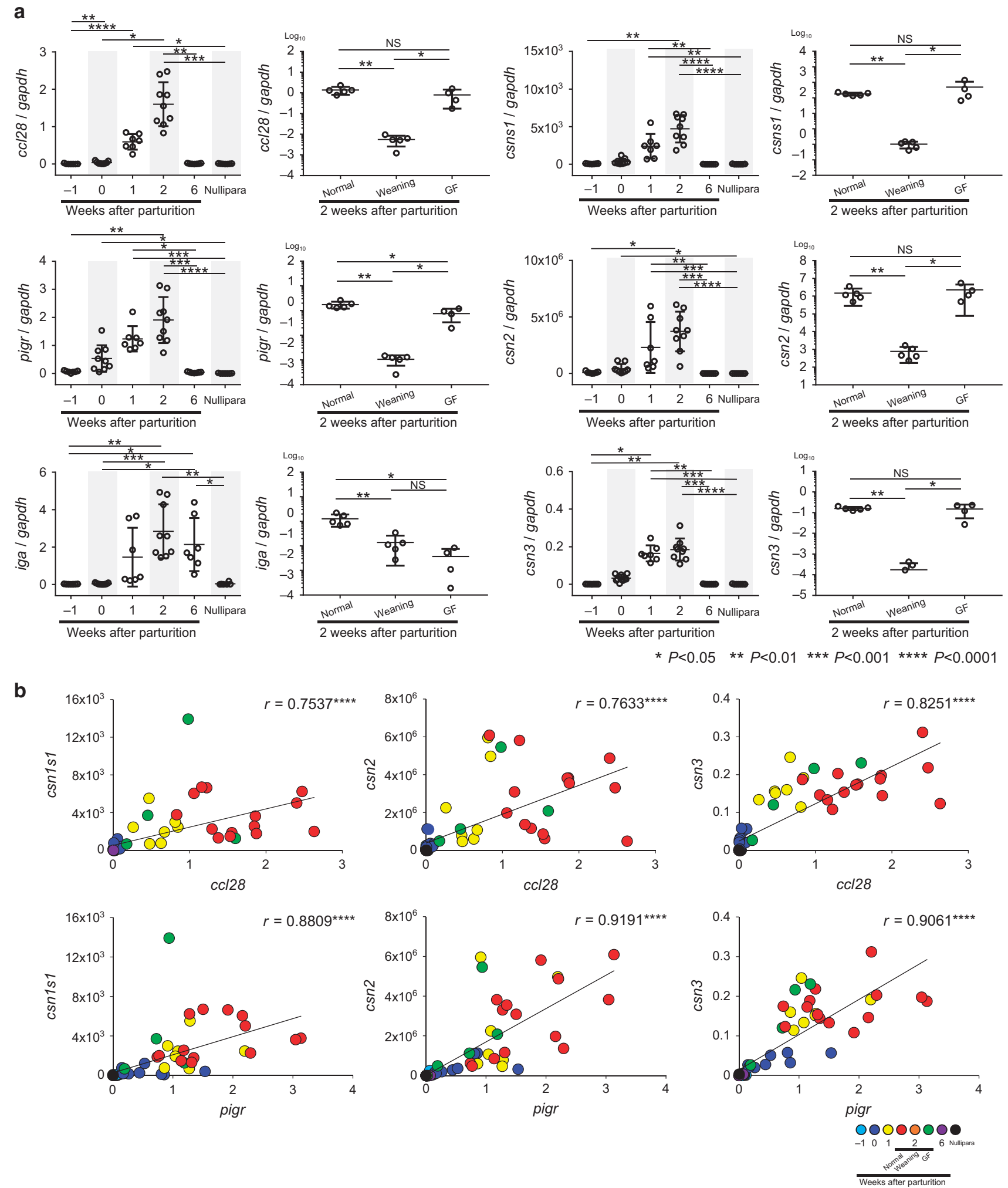

Figure 5 Involvement of the endocrine system in the development of mammary gland immunity. To extract mRNA, mammary glands were harvested at 13 days of gestation $(n=7)$, just after parturition $(n=9)$, and 1 week $(n=7)$, 2 weeks $(n=9)$, and 6 weeks $(n=7)$ after parturition. Tissue was also collected 2 weeks after parturition from mice whose pups were force-weaned 1 week earlier $(n=5)$ and from germ-free mice with pups $(n=4)$ in addition to that from normal mice with pups $(n=5)$. Nonpregnant nulliparous mice were used as a control group $(n=7)$. (a) The expression levels of chemokine (C-C motif) ligand 28 (CCL28), polymeric Ig receptor (plgR), and immunoglobulin A (IgA) gradually increased after the parturition. The levels significantly decreased when pups were weaned, but no differences were observed compared with the respective levels in the germ-free mice with pups. Similarly, expression levels of $\alpha-, \beta-$, and $\kappa$-casein were gradually increased after the parturition. (b) The expression patterns of milk proteins such as $\alpha$-casein, $\beta$ casein, and $\kappa$-casein were similar to those of CCL28 and plgR. Positive correlations were observed upon comparison of the expression levels of CCL28 and plgR with those of $\alpha$-, $\beta$-, and $\kappa$-casein. GF, germ-free mice. ${ }^{\star} P<0.05 ;{ }^{\star \star} P<0.01 ;{ }^{\star \star \star} P<0.001$, ${ }^{\star \star \star \star} P<0.0001$; NS, not significant. 


\section{DISCUSSION}

This study evaluated the microbial community of the mammary gland during lactation. So far, milk samples have been used to demonstrate the presence of microorganisms that are important for neonates to develop not only the intestinal microflora but also the local immune system in the gut. ${ }^{19-21}$ However, it should be emphasized that this is the first report showing the presence of microflora in the mammary gland using genomic DNA extracted from the tissue. We do not exclude the possibility of contamination with DNA of milkderived bacteria present in mammary gland alveoli or the uniformity of extraction efficacy of genomic DNA from various kinds of microorganisms present in the mammary gland; however, our results provide clearer evidence of microbial development in the mammary gland. Most of the microorganisms that we found in the mammary gland are usually present in the gastrointestinal tract (e.g., Bacteroidales and Clostridiales). ${ }^{22}$ The mechanism of bacterial translocation into the mammary gland has not been fully elucidated yet, but recent studies have proposed a new concept suggesting that certain bacteria may translocate into the mammary gland from the maternal gastrointestinal tract through immune cells, such as dendritic cells, via the entero-mammary pathway. ${ }^{23,24}$ Because our results showed that the microbial diversity was already observed in the mammary gland immediately after parturition and was maintained during lactation, it seems reasonable to hypothesize that immune cells present in the maternal gastrointestinal tract acquire homing signals during pregnancy to transport gut microorganisms into the mammary gland. It should be noted that Staphylococcus aureus, ${ }^{25}$ a bacterium capable of causing infectious mastitis in humans and animals, was only found in 1 of the 10 mice analyzed (one of the five mice tested just after parturition), but the frequency was extremely low (4 in 21,332 sequencing reads of $16 \mathrm{~S}$ ribosomal RNA). However, it should be noted that the mammary gland is highly susceptible to infection when a large number of $S$. aureus is exposed via nipples. These results suggest that in a normal condition the microflora developed in the mammary gland may provide environmental pressure against pathogenic bacteria so that they are not included easily in the bacterial community. However, such a microbiological environment breaks occasionally when the tissue is highly infected by pathogens locally.

In the colon, CCL28 expression can be induced by the proinflammatory cytokine interleukin-1 or by bacterial flagellin, as well as by a short-chain fatty acid, butyrate, a product of microbial metabolism. ${ }^{26}$ Butyrate also facilitates the constitutive expression of pIgR in colonic epithelial cells. ${ }^{27}$ This information, coupled with our results showing the presence of the microbiota in the mammary gland, led us to hypothesize that microbial stimulation may also directly or indirectly induce the expression of CCL28 and pIgR in the mammary gland, both of which are essential for the IgA production in milk. Meanwhile, development of the mammary gland is totally controlled by mammotropic hormones associated with pregnancy and lactation. ${ }^{1}$ It is therefore necessary to take into account factors (e.g., hormones) derived from the endocrine system to understand the molecular mechanism of $\operatorname{IgA}$ production in milk. In this study, at 2 weeks after parturition when IgA production sufficiently increases under normal conditions, we collected mammary glands to compare the expression levels of CCL28 and pIgR among three groups: (i) normal mice with pups and a microbiota; (ii) germ-free mice with pups but no microbiota; and (iii) mice after forced weaning, with a microbiota but no pups. We found that suckling-derived stimulation alone drives the expression levels of CCL28 and pIgR, independently of microbiota status, as levels were equivalent between the normal and germ-free mice, but were significantly lower in the mice after forced weaning. More importantly, the expression levels of CCL28 and pIgR and those of three major milk proteins ( $\alpha$-, $\beta$-, and $\kappa$-casein) were positively correlated. These results indicate that the recruitment of IgA-producing plasma cells into the mammary gland via CCL28 and the transportation of IgA into mammary epithelial cells through pIgR are bacteria independent during lactation.

MALTs such as gut-associated lymphoid tissue and nasopharynx-associated lymphoid tissue are important immune structures for T cell-dependent B-cell activation in the mucosal immune system. ${ }^{15,17}$ Once mature naive B cells present in MALTs are activated by antigenic stimulation, they induce the Ig class switching, mostly from IgM to IgA, and migrate into mucosal tissues (e.g., lamina propria) where these activated B cells differentiate into plasma cells to produce IgA. ${ }^{15,17}$ The inguinal lymph node is the draining lymph node of the mammary gland; ${ }^{16}$ however, its function, especially in the IgA production in milk, has not yet been fully elucidated. Using cells individually isolated from the mammary gland and inguinal lymph node, we found a large number of $\mathrm{IgA}^{+} \mathrm{B} 220^{-}$plasma cells in the mammary gland (but not in the inguinal lymph node) 2 weeks after parturition. However, no clear evidence showing the presence of plasmablasts that underwent immunoglobulin class switching from IgM to IgA was observed in the inguinal lymph node throughout the analyzed reproduction cycle. The reason why the inguinal lymph node is not actively involved in the induction of IgA production in milk might be because of the fact that its primary role is to respond to external pathogens (e.g., S. aureus) that infect the mammary gland through a teat opening but are almost undetectable in the mammary gland under normal conditions. Another interesting hypothesis is that the inguinal lymph node might not play a role in the entero-mammary pathway, indicating that it may not pass the inguinal lymph node. In this case, it is impossible to expect that immune cells in the inguinal lymph node would recognize mammary gland residential bacteria that are likely to be derived from the gastrointestinal tract. To this end, our current efforts have been focused on utilizing mice that lack the inguinal lymph node to confirm (i) their susceptibility to S. aureus infection, (ii) microbial diversity in the mammary gland, and (iii) the IgA production in milk.

Recent advances in repertoire analysis have allowed a better understanding of the diversity of the B-cell receptor in various tissues. ${ }^{28}$ Lindner et al. ${ }^{29}$ have demonstrated that the repertoire of IgA in the mammary gland largely mirrored that in the gastrointestinal tract. This makes sense because the 
milk-derived IgA should recognize gut microorganisms in neonates to prevent bacterial infections that cause diarrhea. ${ }^{30}$ Given that IgA-producing plasma cells have been considered to originate from the maternal gastrointestinal tract, ${ }^{23,24}$ it seems reasonable to hypothesize that gut-associated lymphoid tissues such as Peyer's patches, colonic patches, cecal patches, or isolated lymphoid follicles are the most responsible for the production of IgA in milk among the MALTs that initiate immune responses to gut microorganisms. However, we found the gut-like bacterial diversity in the mammary gland, where MALT structures, including mature naive $B$ cells that react to such microorganisms, are absent. This discrepancy might be because of the fact that the bacterial and immune translocation from the maternal gastrointestinal tract to the mammary gland to develop microflora and to produce IgA in the mammary gland, respectively, occur independently. Therefore, our current effort has been focused on understanding the alteration of immune and microbial environments in the mammary gland of antibiotics-treated mice whose mammary gland tissue is ready to recruit IgA-producing cells and transport secreted IgA.

In conclusion, we showed that the development of immunological and bacteriological environments is independently regulated in the mammary gland. Our findings have a significant potential to further research on breastfeeding. Improvement of the quality of the entero-mammary pathway can be an attractive approach to maintaining good physiological conditions for the mammary gland function.

\section{METHODS}

Animals. BALB/c female mice were obtained from Japan SLC (Shizuoka, Japan) and were maintained at the animal facility of the Graduate School of Agricultural Science in Tohoku University (Miyagi Japan). Fundamentally, the mice were used for collecting the mammary gland tissues, inguinal lymph nodes, and Peyer's patches at $-7,0,7,14$, and 42 days (hereafter described as $-1,0,1,2$, and 6 weeks, respectively) after parturition. Some mice were killed 2 weeks after parturition but (i) their pups were removed 1 week before slaughter, (ii) administered freely with a mixture of antibiotics composed of $1 \mathrm{gl}^{-1}$ of ampicillin (Wako), $1 \mathrm{gl}^{-1}$ of neomycin (Wako, Osaka, Japan), and $500 \mathrm{mgl}^{-1}$ of vancomycin (Wako) dissolved in drinking distilled water from 1 week before mating, (iii) exposed with $1 \times 10^{6}$ colony-forming units per $30 \mu \mathrm{l}$ of $S$. aureus (Strain JE2) via nipples of left and right sides of fourth mammary gland under anesthesia with medetomidine hydrochloride (0.3 $\mathrm{mg} \mathrm{kg}^{-1}$, Meiji Seika Pharma, Tokyo, Japan), midazolam (4 $\mathrm{mg} \mathrm{kg}^{-1}$, astellas), and butorphanol tartrate ( $5 \mathrm{mg} \mathrm{kg}^{-1}$, Meiji Seika Pharma) 2 days before slaughter, or (iv) maintained at the germ-free facility of Sankyo Labo Service (Tsukuba, Japan) until slaughter. The stomach contents collected from pups on days 0,7 , and 14 after birth were suspended in phosphate-buffered saline $(1 \mathrm{mg}$ per $10 \mu \mathrm{l})$ to obtain soluble proteins, including immunoglobulins. All experiments with mice were designed in accordance with the protocols approved by the institutional animal care and use committee of the Tohoku University.

Enzyme-linked immunosorbent assay. Concentrations of three immunoglobulin subclasses (IgM, IgG, and IgA) were determined in the supernatants by ELISA. In brief, 96-well ELISA plates (Nunc, Thermo Fisher Scientific, Waltham, MA) were coated overnight at $4{ }^{\circ} \mathrm{C}$ with goat anti-mouse IgM, IgG, or IgA antibodies (all $2 \mu \mathrm{g} \mathrm{ml}^{-1}$, Bethyl Laboratories). After blocking the plates with bovine serum albumin (1\%, Sigma) for $1 \mathrm{~h}$ at room temperature (RT), two serially diluted milk samples were added per plate and incubated for $2 \mathrm{~h}$ at RT. After washing, the plates were treated with horseradish peroxidase-conjugated antimouse IgM, IgG, or IgA antibodies, respectively (all $100 \mathrm{ng} \mathrm{ml}^{-1}$, Bethyl Laboratories, , Montgomery, TX), for $1 \mathrm{~h}$ at RT, and the signals were developed using a tetramethylbenzidine microwell peroxidase substrate system (KPL, SeraCare Life Sciences, Milford, MA). Mouse reference serum (Bethyl Laboratories), containing IgM, IgG, and IgA at known concentrations, was used as a standard.

Histochemistry. Mammary gland tissues collected were fixed in paraformaldehyde (4\%, Nakalai Tesque, Kyoto, Japan) overnight at $4{ }^{\circ} \mathrm{C}$ and embedded in paraffin. Tissue sections $(5 \mu \mathrm{m})$ were dewaxed, then treated with a blocking reagent $(0.5 \%$, PerkinElmer, Waltham, $\mathrm{MA}$ ) for $30 \mathrm{~min}$ at RT, and incubated with a purified rat anti-mouse IgA antibody (500 $\mathrm{ng} \mathrm{ml}^{-1}, \mathrm{C10-3,} \mathrm{BD} \mathrm{Biosciences,} \mathrm{San} \mathrm{Jose,} \mathrm{CA)} \mathrm{or} \mathrm{a}$ purified rat IgG1 isotype control antibody $\left(500 \mathrm{ng} \mathrm{ml}^{-1}, \mathrm{R} 3-34, \mathrm{BD}\right.$ Biosciences) overnight at $4{ }^{\circ} \mathrm{C}$. The sections were then stained with Histofine Simple Stain MAX PO (Rat) (Nichirei Biosciences, Tokyo, Japan) at RT for $1 \mathrm{~h}$, and the signal was developed with $3,3^{\prime}$-diaminobenzidine tetrahydrochloride. Finally, counterstaining with hematoxylin was performed. Some tissue sections collected from the in vivo infection study with $S$. aureus were stained with a purified bovine anti-S. aureus antibody $\left(100 \mu \mathrm{g} \mathrm{ml}^{-1}\right.$, produced in-house) or a purified bovine $\mathrm{IgG}$ control antibody $\left(100 \mu \mathrm{g} \mathrm{ml}^{-1}\right.$, Sigma) overnight at $4{ }^{\circ} \mathrm{C}$ after treatment with the blocking reagent for $30 \mathrm{~min}$ at RT. After washing, the sections were treated with horseradish peroxidaseconjugated sheep anti-bovine IgG antibody $\left(2 \mu \mathrm{g} \mathrm{ml}^{-1}\right.$, Bethyl) at RT for $1 \mathrm{~h}$ and then horseradish peroxidase activity was developed with fluorescein tyramide (PerkinElmer) for $10 \mathrm{~min}$ at RT. After washing, the sections were counterstained with 4',6-diamidino-2-phenylindole (Sigma). Hematoxylin and eosin staining was also performed to investigate the mammary gland development during the experimental period and the pathological alteration when exposed with $S$. aureus. Tissue images were obtained with either BX63 (Olympus, Tokyo, Japan) or BZ-9000 (Keyence, Osaka, Japan).

Real-time PCR. Total RNA was extracted from mammary gland tissues collected 7 days before the parturition and $0,7,14$, and 42 days after the parturition using a ReliaPrep RNA tissue miniprep system (Promega, Madison, WI) according to the manufacturer's protocol. Complementary DNA was synthesized from the total RNA using a PrimeScript RT reagent kit with oligo(dT) primers and random hexamers (Takara, Shiga, Japan). Quantitative PCR was performed using SYBR premix Ex Taq II (Takara) to determine the expression levels of mRNA coding for CCL28, pIgR, IgA, casein- $\alpha$ S1 (CSN1S1), casein- $\beta$ (CSN2), and casein- $\kappa$ (CSN3). The glyceraldehyde-3phosphate dehydrogenase gene (GAPDH) or $\beta$-actin was used as an endogenous control to normalize the expression levels. All primers were designed by the perfect real-time support system (Takara). Data were obtained using a thermal cycle Dice real-time system (Takara)

Flow cytometry. Mammary gland tissues and Peyer's patches collected 7 days before the parturition and $0,7,14$, and 42 days after the parturition were digested with collagenase $\left(1 \mathrm{mg} \mathrm{ml}^{-1}\right.$, Wako) to obtain mononuclear cells. Inguinal lymph nodes harvested from the same mice were processed mechanically to isolate mononuclear cells. The cells were blocked with a purified rat anti-mouse CD16/CD32 antibody $\left(10 \mu \mathrm{g} \mathrm{ml}^{-1}, 2.4 \mathrm{G} 2\right.$, BD Bioscience) at $4{ }^{\circ} \mathrm{C}$ for $15 \mathrm{~min}$ and stained with a phycoerythrin-conjugated rat anti-mouse CD45R/B220 antibody $\left(2 \mu \mathrm{g} \mathrm{ml}^{-1}\right.$, RA3-6B2, BD Bioscience) or phycoerythrinconjugated rat IgG2a isotype control $\left(2 \mu \mathrm{g} \mathrm{ml}^{-1}, \mathrm{R} 35-95, \mathrm{BD}\right.$ Bioscience) at $4{ }^{\circ} \mathrm{C}$ for $30 \mathrm{~min}$. After washing, the cells were fixed in paraformaldehyde (4\%, Nakalai Tesque) at RT for $20 \mathrm{~min}$ and treated with saponin ( $0.1 \%$, Nakalai Tesque) at RT for $15 \mathrm{~min}$. The cells were then incubated with fluorescein isothiocyanate-conjugated rat antimouse IgA antibody (500 $\mathrm{ng} \mathrm{ml}^{-1}, \mathrm{C} 10-3, \mathrm{BD}$ Bioscience) or fluorescein isothiocyanate-conjugated rat IgG1 isotype control (500 $\mathrm{ng} \mathrm{ml}^{-1}, \mathrm{R} 3-34, \mathrm{BD}$ Bioscience) at RT for $30 \mathrm{~min}$ and analyzed using an Accuri C6 flow cytometer (BD Bioscience). A cell viability 
solution (10 $\mu \mathrm{l}$ per analysis, BD Bioscience) was used to exclude dead cells and thus improve the accuracy of the analyses.

Bacterial DNA analyses. Genomic DNA was extracted from the mammary gland at 0 and 14 days after the parturition using a Wizard SV genomic DNA purification system (Promega). The V3 and V4 regions of the bacterial $16 \mathrm{~S}$ ribosomal RNA gene were amplified by PCR using PrimeSTAR HS DNA polymerase (Takara) and the following primers: forward (5'-TGCTCTTCCGATCTGACCCTAC GGGNGGCWGCAG-3') and reverse (5'-CGCTCTTCCGATCTC TGGACTACHVGGGTATCTAATCC-3'), with the adapter tag sequences underlined. The PCR fragments obtained from the first round of PCR were amplified individually in a second round of PCR using the following primers: forward (5'-CAAGCAGAAGACGGC ATACGAGATxxxxxxGTGACTGGAGTTCAGACGTGTGCTCTTC CGATCTGAC- $\left.3^{\prime}\right)$ and reverse $\left(5^{\prime}\right.$-AATGATACGGCGACCACC GAGATCTACACTCTTTCCCTACACGACGCTCTTCCGATCTC TG-3'), including a six-base index, shown as "xxxxxx," to distinguish each sample as described previously. ${ }^{31}$ All PCR products were then sequenced using the MiSeq platform (Illumina, San Diego, CA) with the MiSeq reagent kit v. 3 (600 cycles). The data were analyzed by BaseSpace (Illumina) to identify bacterial species from each sequencing run. A quantitative PCR analysis using the bacteria (tuf gene) quantitative PCR kit (Takara) was performed to determine the copy numbers of bacteria specific gene tuf in extracted DNA samples.

Statistical analysis. Statistical analyses were carried out by one-way analysis of variance with the Kruskal-Walllis test (Figures 1-3 and 5), by comparison analysis of the unpaired two groups using MannWhitney $U$-test (Figure 5) and by a correlation study (Figure 5) using Prism 6 (GraphPad, La Jolla, CA). In addition, QIIME and R were used to compare bacterial diversity (Figure 4). Specifically, a beta_diversity table was constructed by beta_diversity.py (with "-m euclidean" option) and a principal coordinate analysis was executed by principal_coordinates.py in QIIME. Scatter plots were drawn by R.

SUPPLEMENTARY MATERIAL is linked to the online version of the paper at http://www.nature.com/mi

\section{ACKNOWLEDGMENTS}

We thank Dr. Julio Villena for his critical comments regarding this manuscript, Dr. Hidetomo Iwano for his technical advice regarding the in vivo infection model with S. aureus, and Yuki Arino and Takashi Murata for their support at the animal facility of Tohoku University Graduate School of Agricultural Science. Staphylococcus aureus subsp. aureus, Strain JE2, NR-46543 was provided by the Network on Antimicrobial Resistance in Staphylococcus aureus for distribution by BEI Resources, NIAID, NIH. This work was supported by KAKENHI from the Japan Society for the Promotion of Science (16H06207 and 26660245, to T.N.), by the Grant for Joint Research Project of the Institute of Medical Science, the University of Tokyo (to T.N.), by research program on innovative technologies for animal breeding, reproduction and vaccine development from the Ministry of Agriculture, Forestry and Fisheries of Japan (to T.N. and H.A.), and by Coreto-Core Program (Advanced Research Networks) from the Japan Society for the Promotion of Science (Establishment of international agricultural immunology research-core for a quantum improvement in food safety, to Ha.K., W.K., T.N., and H.A.).

\section{AUTHOR CONTRIBUTIONS}

K.N., K.U., and T.N. planned and conducted the experiments, analyzed data, and wrote the paper. Y.F., M.A., M.F. Y.Su., Y.Sa., M.K., N.S., E.J.P., S.S. H.Y., and K.W. conducted the experiments. Hi.K. Ha.K., and H.A., wrote the paper.

\section{DISCLOSURE}

The authors declared no conflict of interest.

(c) 2018 Society for Mucosal Immunology

\section{REFERENCES}

1. Brisken, C. \& Ataca, D. Endocrine hormones and local signals during the development of the mouse mammary gland. Wiley Interdiscip. Rev. Dev. Biol. 4, 181-195 (2015).

2. Brandtzaeg, P. The secretory immune system of lactating human mammary glands compared with other exocrine organs. Ann. NY Acad. Sci. 409, 353-382 (1983).

3. Faucette, A.N., Unger, B.L., Gonik, B. \& Chen, K. Maternal vaccination: moving the science forward. Hum. Reprod. Update 21, 119-135 (2015).

4. Hurley, W.L. \& Theil, P.K. Perspectives on immunoglobulins in colostrum and milk. Nutrients 3, 442-474 (2011).

5. Brandtzaeg, P. The mucosal immune system and its integration with the mammary glands. J. Pediatr. 156, S8-15 (2010).

6. Wilson, E. \& Butcher, E.C. CCL28 controls immunoglobulin (lg)A plasma cell accumulation in the lactating mammary gland and IgA antibody transfer to the neonate. J. Exp. Med. 200, 805-809 (2004).

7. Morteau, O. et al. An indispensable role for the chemokine receptor CCR10 in IgA antibody-secreting cell accumulation. J. Immunol. 181, 6309-6315 (2008).

8. Mostov, K.E. Transepithelial transport of immunoglobulins. Annu. Rev. Immunol. 12, 63-84 (1994)

9. van der Feltz, M.J., de Groot, N., Bayley, J.P., Lee, S.H., Verbeet, M.P. \& de Boer, H.A. Lymphocyte homing and Ig secretion in the murine mammary gland. Scand. J. Immunol. 54, 292-300 (2001).

10. Fernandez, L. et al. The human milk microbiota: origin and potential roles in health and disease. Pharmacol. Res. 69, 1-10 (2013).

11. Martin, V. et al. Sharing of bacterial strains between breast milk and infant feces. J. Hum. Lact. 28, 36-44 (2012).

12. West, P.A., Hewitt, J.H. \& Murphy, O.M. Influence of methods of collection and storage on the bacteriology of human milk. J. Appl. Bacteriol. 46, 269277 (1979).

13. Vorbach, C., Capecchi, M.R. \& Penninger, J.M. Evolution of the mammary gland from the innate immune system?. Bioessays 28, 606-616 (2006).

14. Boumahrou, N., Chevaleyre, C., Berri, M., Martin, P., Bellier, S. \& Salmon, $\mathrm{H}$. An increase in milk IgA correlates with both plgR expression and IgA plasma cell accumulation in the lactating mammary gland of PRM/Alf mice. J. Reprod. Immunol. 96, 25-33 (2012).

15. Kiyono, H. \& Fukuyama, S. NALT- versus Peyer's-patch-mediated mucosal immunity. Nat. Rev. Immunol. 4, 699-710 (2004).

16. Sheng, F., Inoue, Y., Kiryu, S., Watanabe, M. \& Ohtomo, K. Lymph drainage from the mammary glands in mice: a magnetic resonance lymphographic study with gadofluorine M. Acad. Radiol. 18, 512-517 (2011).

17. Kunisawa, J., Kurashima, Y. \& Kiyono, H. Gut-associated lymphoid tissues for the development of oral vaccines. Adv. Drug Deliv. Rev. 64, 523-530 (2012).

18. Tanaka, Y., Takahashi, H., Simidu, U. \& Kimura, B. Design of a new universal real-time PCR system targeting the tuf gene for the enumeration of bacterial counts in food. J. Food Prot. 73, 670-679 (2010).

19. Martin, R. et al. Human milk is a source of lactic acid bacteria for the infant gut. J. Pediatr. 143, 754-758 (2003).

20. Martin, R. et al. Isolation of bifidobacteria from breast milk and assessment of the bifidobacterial population by PCR-denaturing gradient gel electrophoresis and quantitative real-time PCR. Appl. Environ. Microbiol. 75, 965969 (2009).

21. Albesharat, R., Ehrmann, M.A., Korakli, M., Yazaji, S. \& Vogel, R.F. Phenotypic and genotypic analyses of lactic acid bacteria in local fermented food, breast milk and faeces of mothers and their babies. Syst. Appl. Microbiol. 34, 148-155 (2011).

22. Monteagudo-Mera, A., Arthur, J.C., Jobin, C., Keku, T., Bruno-Barcena J.M. \& Azcarate-Peril, M.A. High purity galacto-oligosaccharides enhance specific Bifidobacterium species and their metabolic activity in the mouse gut microbiome. Benef. Microbes 7, 247-264 (2016).

23. Rodriguez, J.M. The origin of human milk bacteria: is there a bacterial entero-mammary pathway during late pregnancy and lactation?. Adv. Nutr. 5, 779-784 (2014).

24. Jost, T., Lacroix, C., Braegger, C.P., Rochat, F. \& Chassard, C. Vertical mother-neonate transfer of maternal gut bacteria via breastfeeding. Environ. Microbiol. 16, 2891-2904 (2014).

25. Niebyl, J.R., Spence, M.R. \& Parmley, T.H. Sporadic (nonepidemic) puerperal mastitis. J. Reprod. Med. 20, 97-100 (1978). 
26. Ogawa, H., limura, M., Eckmann, L. \& Kagnoff, M.F. Regulated production of the chemokine CCL28 in human colon epithelium. Am. J. Physiol. Gastrointest. Liver Physiol. 287, G1062-G1069 (2004).

27. Kvale, D. \& Brandtzaeg, P. Constitutive and cytokine induced expression of HLA molecules, secretory component, and intercellular adhesion molecule-1 is modulated by butyrate in the colonic epithelial cell line HT-29. Gut 36, 737-742 (1995).

28. Lee, J. et al. Molecular-level analysis of the serum antibody repertoire in young adults before and after seasonal influenza vaccination. Nat. Med. 22, 1456-1464 (2016).
29. Lindner, C. et al. Diversification of memory B cells drives the continuous adaptation of secretory antibodies to gut microbiota. Nat. Immunol. 16, 880-888 (2015).

30. Rogier, E.W. et al. Secretory antibodies in breast milk promote long-term intestinal homeostasis by regulating the gut microbiota and host gene expression. Proc. Natl. Acad. Sci. USA 111, 3074-3079 (2014).

31. Suyama, Y. \& Matsuki, Y. MIG-seq: an effective PCR-based method for genome-wide single-nucleotide polymorphism genotyping using the nextgeneration sequencing platform. Sci. Rep. 5, 16963 (2015). 